\title{
Efficacy and Safety of HSK3486 for Anesthesia/Sedation in Patients Undergoing Fiberoptic Bronchoscopy: A Multicenter, Double-Blind, Propofol-Controlled, Randomized, Phase 3 Study
}

\author{
Zhen Luo ${ }^{1} \cdot$ Hong Tu ${ }^{1} \cdot$ Xiang Zhang $^{1} \cdot$ Xiao Wang $^{1} \cdot$ Wen Ouyang $^{2} \cdot$ Xinchuan Wei $^{3} \cdot$ Xiaohua Zou $^{4} \cdot$ Zhaoqiong Zhu $^{5}$. \\ Yalan $\mathrm{Li}^{6} \cdot$ Wangning Shangguan ${ }^{7} \cdot \mathrm{Hui} \mathrm{Wu}^{8} \cdot$ Yaping Wang $^{9} \cdot$ Qulian Guo $^{10}$
}

Accepted: 12 December 2021 / Published online: 14 February 2022

(c) The Author(s) 2022

\begin{abstract} bronchoscopy. assessments and drug concentrations were also measured. group. HSK3486 had a similar terminal elimination half-life as propofol.

\section{Introduction}

Fiberoptic bronchoscopy has gradually become an important diagnostic and therapeutic procedure for the treatment of respiratory diseases, which has been enhanced by the use of a laryngeal mask airway (LMA) method that improves airway support and stable oxygen saturation, with a convenient port of entry during fiberoptic bronchoscopy [1]. Sedation was initially rarely used in fiberoptic bronchoscopy because there was a concern about adverse events (AEs) [2]. As this procedure can produce great discomfort in patients and elicit
\end{abstract}

Background Fiberoptic bronchoscopy is a complex procedure with the need for sufficient patient anesthesia/sedation while maintaining safety. This trial aimed to evaluate the efficacy, safety, and pharmacokinetics of HSK3486 during fiberoptic

Methods This multicenter, double-blind, randomized, non-inferiority, parallel-group phase 3 trial was conducted in patients who underwent fiberoptic bronchoscopy. Patients randomly received HSK3486 $0.4 \mathrm{mg} / \mathrm{kg}(N=134)$ or propofol $2.0 \mathrm{mg} / \mathrm{kg}(N$ =133). The primary efficacy endpoint was the successful rate of fiberoptic bronchoscopy, and secondary efficacy endpoints included successful induction of anesthesia/sedation, duration, time to being fully alert, and time to patient discharge. Safety

Results A total of 267 patients completed fiberoptic bronchoscopy, with a success rate of $100 \%$ and a $95 \%$ confidence interval of -2.8 to $2.8 \%$ for the difference between the groups, which met the predesigned criteria of $>-8 \%$, confirming the noninferiority of anesthesia/sedation produced by HSK3486 compared to propofol. Among the secondary efficacy endpoints, only time to full alertness (median 8.50 vs. $6.00 \mathrm{~min}, P=0.012$ ) and time to discharge (median 13.00 vs. $9.87 \mathrm{~min}, P=0.002$ ) were slightly longer in the HSK3486 group. The incidence of adverse events was significant lower in the HSK3486 group (52.6 vs. $76.5 \%, P<0.001)$ mainly because of less pain on injection ( 4.4 vs. $39.4 \%, P<0.001)$ compared to the propofol

Conclusions HSK3486 exhibited non-inferiority anesthesia/sedation compared to propofol in patients undergoing fiberoptic bronchoscopy, and had a good safety profile with a lower incidence of pain on injection.

Trial Registration Clinicaltrials.gov, NCT04111159, registered on 1 October 2019

Zhen Luo and Hong Tu contributed equally to this article.

Xiao Wang

1490829116@qq.com

Extended author information available on the last page of the article

\section{Key Points}

This multicenter, double-blind, randomized, noninferiority, parallel-group phase 3 trial was conducted to evaluate the efficacy, safety, and pharmacokinetics of HSK3486 in patients undergoing fiberoptic bronchoscopy.

Patients who received HSK3486 completed fiberoptic bronchoscopy with a success rate of $100 \%$ and showed non-inferiority anesthesia/sedation characteristics compared to propofol during fiberoptic bronchoscopy.

HSK3486 exhibited a good safety profile with a lower incidence of pain on injection. 
a high incidence of hypoxia, sedation has gradually been developed for painless bronchoscopy [3-5]. Subsequent studies have shown that sedation leads to better tolerance [6] and higher physician satisfaction for the procedure [7]. Therefore, to ensure the safety and comfort of patients while meeting the operational requirements of sharing the airway is a major challenge for the successful implementation of painless fiberoptic bronchoscopy [5, 8].

The British Thoracic Society states that patients undergoing fiberoptic bronchoscopy should be sedated when there are no contraindications [9] to facilitate patient comfort and alleviate anxiety, coughing, and dyspnea, while reducing the complications of the procedure $[6,10,11]$. Previous studies have shown that propofol is often used to induce moderate sedation or general anesthesia for a fiberoptic bronchoscopy procedure because of its rapid onset, fast recovery, high clearance rate, and good tolerance [12-14]. There is improved patient perception of sedation and procedure tolerance, and an overall reduction in cough and the sensation of asphyxiation when propofol is administered [6]. Propofol used as monotherapy for sedation does not result in analgesia, so it is often combined with opiates or local anesthetics to improve patient tolerance [14, 15], However, propofol has a number of known limitations [16, 17], such as a narrow therapeutic window, high incidence of hypotension, respiratory depression, pain on injection, and a lack of availability of antagonists.

HSK3486 (Trade name: ciprofol, Haiso Pharmaceutical Group Co., Ltd, Chengdu, China), a novel 2,6-disubstituted phenol derivative, binds more effectively to the gammaaminobutyric acid-A $\left(\mathrm{GABA}_{\mathrm{A}}\right)$ receptor than does propofol [18]. After initial evaluations of absorption, distribution, metabolism, and excretion (ADME) processes [19], the maximum concentration $\left(C_{\max }\right)$, time to $C_{\max }\left(t_{\max }\right)$, terminal elimination half-life $\left(t_{1 / 2}\right)$, and mean residence time (MRT) were similar between HSK3486 and propofol, while clearance (CL), apparent volume of distribution $\left(V_{\mathrm{d}}\right)$ and apparent volume of distribution at steady state $\left(V_{\mathrm{ss}}\right)$ were different in a phase 1 trial [20]. In addition, a study on age-related effects of HSK3486 revealed that a dose of $0.3 \mathrm{mg} / \mathrm{kg}$ was similarly efficacious in the elderly compared with $0.4 \mathrm{mg} / \mathrm{kg}$ in nonelderly patients [21].

Phase 2 [22] and phase 3 trials (clinicaltrials.gov, NCT03674008, on submission) for gastrointestinal endoscopy procedures demonstrated the efficacy and safety of HSK3486 in patients undergoing endoscopy. The duration of anesthesia/sedation in patients undergoing fiberoptic bronchoscopy was similar to that of gastrointestinal endoscopy, which is generally $10-30 \mathrm{~min}$ and the drug administration methods are also similar, which is an initial intravenous (i.v.) bolus injection followed by top-up doses if required. Therefore, this multicenter, double-blind, propofol-controlled, randomized, phase 3 trial was carried out to evaluate the efficacy and safety of HSK3486 in patients undergoing fiberoptic bronchoscopy.

\section{Material and Methods}

\subsection{Study Design and Procedure}

This multicenter, double-blind, randomized, propofol-controlled, non-inferiority prospective phase 3 trial was conducted in ten study centers in China between 6 December 2019 and 19 June 2020 in accordance with the Declaration of Helsinki. The study was approved by the ethics committee of each participating hospital. The trial was prospectively registered at clinicaltrials.gov (NCT04111159). Written informed consent was obtained from all patients before they were enrolled.

The intention of the trial was to enroll consecutively 260 eligible patients from each study center and randomly allocate them to a HSK3486 $0.4 \mathrm{mg} / \mathrm{kg}$ group or a propofol $2.0 \mathrm{mg} / \mathrm{kg}$ group in a ratio of $1: 1$. The duration of the trial included the screening period (within 7 days before fiberoptic bronchoscopy), baseline period (the same day of fiberoptic bronchoscopy), the observation period drug postadministration and the follow-up post-procedure period (within 2-4 days post fiberoptic bronchoscopy).

The patients enrolled were aged between 18 and 80 years, male or female, with American Society of Anesthesiologists (ASA) grade I-III, and were to receive diagnostic and/or therapeutic fiberoptic bronchoscopy LMA assisted. Exclusion criteria included patients allergic to egg and soybean products, propofol, opioids, and their antidotes. Patients with contraindications to deep sedation/general anesthesia, or with a history of previous anesthesia/sedation accidents, or who had undergone endotracheal intubation and/ or mechanical ventilation prior to fiberoptic bronchoscopy, were also excluded. Detailed inclusion and exclusion criteria are presented in the Electronic Supplementary Material (ESM) Table 1.

Patients had baseline inclusion/exclusion criteria, weight, vital signs, blood samples, pregnancy tests (urine human chorionic gonadotropin for child-bearing aged female patients), 12-lead electrocardiogram (ECG), and Modified Observer Assessment of Alertness/Sedation (MOAA/S) scores measured on the day of the procedure. Eligible patients inhaled $2 \%$ lidocaine $(10 \mathrm{~mL})$ delivered by atomization within $1 \mathrm{~h}$ before the bolus doses of HSK3486 or propofol were i.v. injected. Supplementary oxygen was administered at a flow rate of $>6 \mathrm{~L} / \mathrm{min}$ at least $3 \mathrm{~min}$ before the first study dose was administered and until patients were fully alert (the first time of MOAA/S $=5$ for three consecutive measurements). 
All the researchers were divided into a blinded group and an un-blinded group and the administrations of study drugs were obscured. The anesthesiologist gave instructions and an investigating nurse of the un-blinded group administered the study drugs. Patients aged $<65$ years were given $0.2 \mu \mathrm{g} / \mathrm{kg}$ sufentanil i.v. in advance and the initial dose of HSK3486 or propofol was then injected i.v. after 3 min ( \pm 5 s). Previous phase 2 [22] and phase 3 (NCT03674008, on submission) studies on sedation for gastrointestinal endoscopy procedures demonstrated that the initial dose of 0.4 $\mathrm{mg} / \mathrm{kg}$ HSK3486 was comparable to $2.0 \mathrm{mg} / \mathrm{kg}$ propofol. Because of the similar duration and administration method during gastrointestinal endoscopy procedures, the HSK3486 $0.4 \mathrm{mg} / \mathrm{kg}$ and positive control propofol $2.0 \mathrm{mg} / \mathrm{kg}$ doses were also selected for patients undergoing fiberoptic bronchoscopy in the present trial. The infused volumes of both drugs were similar, comprising $0.4 \mathrm{mg} / \mathrm{kg}$ with a dilution of $2.5 \mathrm{mg} / \mathrm{mL}=0.16 \mathrm{~mL} / \mathrm{kg}$ for HSK3486 and $2 \mathrm{mg} / \mathrm{kg}$ with a dilution of $10 \mathrm{mg} / \mathrm{mL}=0.2 \mathrm{~mL} / \mathrm{kg}$ for propofol. HSK3486 was initially administered i.v. at a dose of $0.4 \mathrm{mg} / \mathrm{kg}$ for patients aged $<65$ years, with top-up doses of $0.15 \mathrm{mg} / \mathrm{kg}$ during the induction and maintenance phases if required. Similarly, propofol was injected i.v. at $2.0 \mathrm{mg} / \mathrm{kg}$ in patients aged $<65$ years, with top-up doses of $0.75 \mathrm{mg} / \mathrm{kg}$ if required. Top-up doses were allowed when inserting the laryngeal mask and the fiberoptic bronchoscope, otherwise at intervals $\geq 2$ min and at most five times during any 15 -min window, with propofol being the alternative rescue sedative. If sedation was still insufficient, sufentanil $(0.05-0.1 \mu \mathrm{g} / \mathrm{kg})$ was given up to a maximum dose of $0.4 \mu \mathrm{g} / \mathrm{kg}$. Patients aged $\geq 65$ years had their doses of sufentanil and the study medication reduced by $25 \%$.

The laryngeal mask (LM), which is routinely used in our hospitals, was inserted when the MOAA/S score was $\leq 1$ and a swivel adapter was connected to its breathing circuit. Then the bronchoscope was inserted through a tunnel inside the LM and pushed into the trachea. Physical monitoring including continuous electrocardiography and pulse oximetry, and automated noninvasive blood pressure was recorded every $2 \mathrm{~min}$. Vital signs and adverse events (AEs) were continuously measured and recorded during the procedure. The MOAA/S score was recorded within $30 \mathrm{~s}$ once the fiberoptic bronchoscope was extracted and then at 1-min intervals until the patient was fully alert, followed by the modified Aldrete score, assessed every 2 min until the score was $\geq 9$ for three consecutive measurements. A satisfaction evaluation scale that ranged from 0 to 10 (sum of five items) was used for patients and anesthesiologists before discharge, with a higher score indicating better satisfaction (ESM Table 2).

\subsection{Efficacy Assessments}

\subsubsection{Primary Efficacy Outcomes}

The success rate of fiberoptic bronchoscopy was assessed according to the following criteria: (1) completion of fiberoptic bronchoscopy; (2) no requirement for any alternative sedative/anesthetic, which means the top-up doses of the experimental drugs were administered no more than five times in any 15-min window from the initial administration to the completion of the procedure.

\subsubsection{Secondary Efficacy Outcomes}

(1) Time to successful induction of anesthesia/sedation, defined as the time from the start of study drug administration to MOAA/S $\leq 1 ;$ (2) time to being fully alert, defined as the time from the fiberoptic bronchoscope extraction or/ and the time from the last drug administration to a MOAA/S score of 5 for three consecutive measurements; (3) time to discharge, defined as the time from the fiberoptic bronchoscope extraction or/and the time from the last drug administration to the initial appearance of three consecutive Aldrete measurements of 9 ; (4) the top-up frequency and dosage of the study medications and sufentanil; (5) anesthesia/sedation satisfaction scores of patients and anesthesiologists collected when patients were ready for discharge.

\subsection{Safety Assessments}

AEs were evaluated for frequency, severity, association to the study drug, relationship to the procedure, and outcomes. The severity of AEs was graded based on the Common Terminology Criteria for Adverse Events (CTCAEs, version 5.0) [23]. The causal relationship of an $\mathrm{AE}$ to the investigated drug (HSK3486 or propofol) was assessed by the investigator (or a medically qualified assistant) using the classifications provided in ESM Table 3. AEs were considered to be severe if a patient's daily function was interfered with or their life was threatened. If cough, physical activity, bronchospasm, wheezing, or other reactions caused by operation irritation were observed in patients, they were recorded as operational reactions of fiberoptic bronchoscopy. Sedation-related AEs, including hypoxia, hypotension, and bradycardia, were evaluated from the time of administration of bolus doses of the study drug until the discharge of patients. Hypoxia was defined as pulse oxygen saturation $\left(\mathrm{SpO}_{2}\right)<90 \%$ lasting for $>30 \mathrm{~s}$; hypotension was defined as systolic blood pressure (SBP) $<90 \mathrm{mmHg}$ or a $20 \%$ decrease from baseline lasting for $>2 \mathrm{~min}$; bradycardia was defined as a heart rate $(\mathrm{HR})<50$ beats/min and lasting for $>2 \mathrm{~min}$. Laboratory tests (routine blood/urine and blood 
biochemistry), vital signs, and 12-lead ECG recordings were also assessed throughout the study.

\subsection{Blood Sampling and Plasma Concentration}

A phase 1 study conducted in Chinese healthy subjects found that the plasma concentration of HSK3486 had a threephase elimination characteristic and the $t_{\max }$ was 2 min after administration [24]. Thus, four venous blood samples ( $3 \mathrm{~mL})$ were collected within $1 \mathrm{~h}$ before the initiation of lidocaine atomization inhalation, $2 \mathrm{~min} \pm 10 \mathrm{~s}$ after the initial administration of the study drug, and at approximately $10 \mathrm{~min}$ and within 1-6 $\mathrm{h}$ after extraction of the fiberoptic bronchoscope. Plasma concentrations of HSK3486 and propofol were measured by validated liquid chromatography tandem mass spectrometry (LC-MS/MS) with a low limit of quantification (LLOQ) of $5 \mathrm{ng} / \mathrm{mL}$. A plasma concentration below LLOQ was recorded as below the quantitation limit (BQL) [21].

\subsection{Statistical Analysis}

Statistical analyses were carried out using SAS ver. 9.4 (SAS Institute Inc, Cary, NC, US). The study was designed as a non-inferiority study with a non-inferiority margin of $8 \%$ with respect to the success rate [25-28]. At a power of $80 \%$, a one-sided type I error rate of 0.025 and assuming that the success rates of fiberoptic bronchoscopy after HSK3486 and propofol administration were both $95 \%$, a total of 260 patients needed to be enrolled in the study, considering an anticipated dropout rate of $10 \%$, with 130 patients in each treatment arm.

For the primary efficacy outcome, the difference of success rates of fiberoptic bronchoscopy between HSK3486 and propofol (HSK3486 - propofol) and the corresponding two-sided 95\% confidence intervals (CIs) were calculated. Non-inferiority between HSK3486 and propofol was concluded if the lower boundary of the $95 \% \mathrm{CI}$ of the difference (HSK3486-propofol) of the success rate was $>-8 \%$. The success rates of fiberoptic bronchoscopy were compared between the HSK3486 and propofol groups using Fisher's exact method adjusted for age-stratification factors. For comparison of secondary efficacy outcomes, the Cochran-Mantel-Haenszel (CMH) test or Fisher's exact probability test was used to compare the categorical variables between two groups, while a $t$-test or Wilcoxon rank sum test was used to compare continuous variables. The incidence of AEs and drug-related AEs between the two groups was compared using a chi-squared test or Fisher's exact probability test. Continuous variables are presented as the mean \pm standard deviation (SD) or median with range (minimum, maximum), while categorical variables are given as numbers with percentages. The study was designed to prove the non-inferiority between HSK3486 and propofol, with respect to success rate (primary endpoint), and all other comparisons were only of an exploratory nature (all $P$-values are two-sided and were not adjusted for comparisons). The full analysis set (FAS) included all patients randomly assigned to receive at least one drug dose (HSK3486 or propofol) after randomization according to the intention to-treat (ITT) principle and at least one evaluable efficacy outcome was used for analysis of all efficacy outcomes and baseline characteristics of patients. The safety set (SS) and pharmacokinetics analysis set (PKS) included all patients based on the "as treated" principle, was used for the analysis of safety outcomes and plasma concentration measurements, respectively.

\section{Results}

\subsection{Patients' Disposition and Baseline Characteristics}

A total of 302 patients were screened, of whom 267 met the eligible criteria, and were randomized to receive HSK3486 $(n=134)$ or propofol $(n=133)$ (Fig. 1). Thus, all efficacy outcomes were analyzed in the FAS (134 vs. 133, ITT). Due to one patient being randomized to the propofol group initially, but who inadvertently was given HSK3486, there were 135 patients in the HSK3486 group and 132 patients in the propofol group for safety and pharmacokinetic outcome (135 vs. 132, as treated) measurements. Overall, the demographic and baseline characteristics were similar between the two groups (Table 1). Of the patients, $49.4 \%$ were female and $89.1 \%$ were aged $<65$ years. All patients had a MOAA/S score of 5 at baseline and $263(98.5 \%)$ patients were ASA grade I-II.

\subsection{Efficacy}

\subsubsection{Primary Efficacy Outcome}

All patients completed the fiberoptic bronchoscopy and no patient received alternative sedative/anesthetic drugs, with success rates of $100 \%$ in both the HSK3486 and the propofol groups. The 95\% CI of the difference (HSK3486- propofol) of success rates was -2.8 to $2.8 \%$, which met the predesigned criteria of $>-8 \%$, indicating that the non-inferiority of HSK3486 compared to propofol with respect to the success rate was confirmed (Table 2).

\subsubsection{Secondary Efficacy Outcomes}

The time course of the MOAA/S scores in the two groups is shown in Fig. 2. Table 3 summarizes the secondary efficacy endpoints of patients in the HSK3486 and propofol groups. The median duration of fiberoptic bronchoscopy, 
Fig. 1 Enrolment flow diagram. $F A S$ full analysis set, $P K S$ pharmacokinetics analysis set, SS safety set

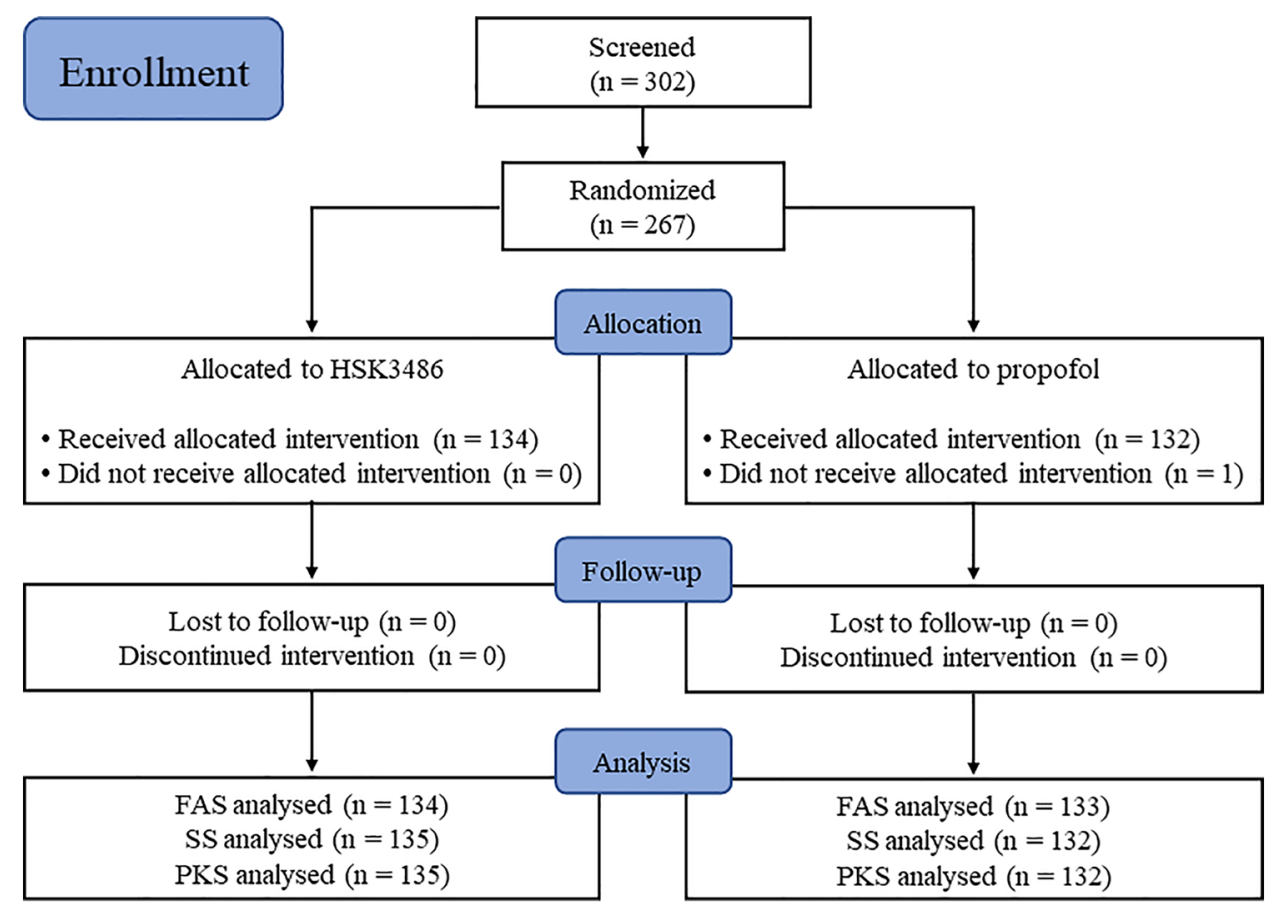

median insertion duration, and the median time to successful induction of anesthesia/sedation were similar in the two groups (all $P>0.05$ ). Patients in the HSK3486 group took a longer time to regain full alertness and to be discharged (all $P<0.05$ ). More than half of the patients in both groups completed fiberoptic bronchoscopy without requiring top-up dosing, and the median times of top-up doses were nearly the same between the two groups. HSK3486 produced comparable satisfaction scores for both patients $(P=0.341)$ and anesthesiologists $(P=0.972)$ compared to the propofol group. Three patients in each group received on one occasion a top-up dose of sufentanil, due to cough and/or body movements during the procedure.

\subsection{Safety}

A total of $172(64.4 \%)$ patients experienced 286 AEs. There were no $\geq$ grade 3 AEs in the HSK3486 group, all being grade 1 or grade 2. Most AEs were self-curing and selflimited. No patients withdrew from the study because of AEs and no serious adverse events (SAEs) occurred (Table 4).

A total of 66 drug-related AEs occurred in 50 (37.0\%) patients treated with HSK3486 and 123 occurred in 93 (70.5\%) patients treated with propofol $(P<0.001)$. The higher incidence of AEs in the propofol group can be mainly attributed to the higher incidence of pain on injection $(P<$ 0.001) (Table 5).

Sedation-related AEs occurred in the two groups, being $40(29.6 \%)$ and $42(31.8 \%)$, respectively $(P=0.698)$ (Table 4). The incidence of hypoxia was a little higher in the HSK3486 patient group than that in the propofol group, but the duration was similar in the two groups. The incidence of hypotension and mean duration in the HSK3486 group were lower than in the propofol group. Although the mean duration of bradycardia was slightly longer in the HSK3486 group, the incidence of bradycardia was similar to that in the propofol group. AEs related to operant reaction of fiberoptic bronchoscopy occurred in both groups, with three patients (one case of wheezing and two cases of bronchospasm) in the HSK3486 group and four patients (two cases of bronchospasm and two cases of body movement) in the propofol group (Table 4).

Vital signs of patients in the HSK3486 and propofol groups during the procedure are presented in Fig. 3. The blood pressure of both groups showed a downward trend 4 min after initial drug administration, with a decrease range within $10.92 \%$, which gradually recovered to the baseline value. HSK3486 induced a smaller decreasing trend in mean arterial blood pressure (MAP) from baseline than did propofol $(P=0.002)$, with maximum mean percentage decreases of $-20.00 \%$ (SD $10.99 \%$ ) and -24.00 (SD 9.91\%), respectively. The respiratory rate in both groups showed a decreasing trend within 2 min of drug administration. HR in both groups fluctuated around baseline after administration, with a maximum fluctuation of $10 \%$, while $\mathrm{SpO}_{2}$ changed slightly after administration, within a range of $1.88 \%$. Laboratory tests and 12-lead ECG recordings showed no clinically meaningful differences. 
Table 1 Demographic and baseline characteristics of patients (full analysis set)

\begin{tabular}{|c|c|c|c|}
\hline & $\operatorname{HSK} 3486(n=134)$ & Propofol $(n=133)$ & $P$-value \\
\hline Age, years & $46.60 \pm 15.31$ & $46.90 \pm 13.98$ & 0.849 \\
\hline Age range, $n(\%)$ & & & 1.000 \\
\hline$\geq 18$ and $<65$ years & $119(88.8)$ & $119(89.5)$ & \\
\hline$\geq 65$ and $<80$ years & $15(11.2)$ & $14(10.5)$ & \\
\hline Gender, $n(\%)$ & & & 0.159 \\
\hline Male & $62(46.3)$ & $73(54.9)$ & \\
\hline Female & $72(53.7)$ & $60(45.1)$ & \\
\hline Height (m) & $1.61 \pm 0.08$ & $1.63 \pm 0.08$ & 0.073 \\
\hline Weight (kg) & $60.60 \pm 9.47$ & $61.13 \pm 9.18$ & 0.642 \\
\hline $\operatorname{BMI}\left(\mathrm{kg} / \mathrm{m}^{2}\right)$ & $23.20 \pm 2.64$ & $22.92 \pm 2.60$ & 0.374 \\
\hline ASA classification, $n(\%)$ & & & 0.151 \\
\hline Grade I & $63(47.0)$ & $50(37.6)$ & \\
\hline Grade II & $68(50.7)$ & $82(61.7)$ & \\
\hline Grade III & $3(2.2)$ & $1(0.8)$ & \\
\hline MOAA/S & $5.0(0.00)$ & $5.0(0.00)$ & 1.000 \\
\hline Diagnostic and therapeutic category, $n(\%)$ & & & 1.000 \\
\hline General diagnostic & $122(91.0)$ & $121(91.0)$ & \\
\hline Advanced diagnostic & $10(7.5)$ & $10(7.5)$ & \\
\hline Therapeutic & $2(1.5)$ & $2(1.5)$ & \\
\hline Modified Mallampati score, $n(\%)$ & & & 0.417 \\
\hline Grade I & $90(67.2)$ & $83(62.4)$ & \\
\hline Grade II & $44(32.8)$ & $50(37.6)$ & \\
\hline
\end{tabular}

Data are given as the mean \pm SD or numbers with percentages in parentheses

$A S A$ American Society of Anesthesiologists, $B M I$ body mass index, MOAA/S Modified Observer Assessment of Alertness/Sedation, $S D$ Standard deviation

Table 2 Achievement of fiberoptic bronchoscopy (full analysis set)

\begin{tabular}{lll}
\hline & HSK3486 $(n=134)$ & Propofol $(n=133)$ \\
\hline Completion of fiberoptic bronchoscopy & 134 & 133 \\
Success rates of fiberoptic bronchoscopy & $100.0 \%$ & $100.0 \%$ \\
Difference (HSK3486-propofol) & 0.0 & $(-2.8 \%, 2.8 \%)$ \\
$95 \%$ CI & & \\
\hline
\end{tabular}

CI confidence interval

\subsection{Plasma Concentration}

HSK3486 had a similar terminal elimination half-life to propofol. The average plasma concentrations of both drugs reached maximal values at $2 \min \pm 10 \mathrm{~s}$ after initial administration, and decreased rapidly between $10 \mathrm{~min}$ and $6 \mathrm{~h}$ after extraction of the fiberoptic bronchoscope. The plasma concentration during the first $6 \mathrm{~h}$ followed first-order kinetics (Fig. 4).

\section{Discussion}

Various classes of anesthetic drugs have been used in fiberoptic bronchoscopy, such as benzodiazepines and opioids, or fospropofol and dexmedetomidine. Benzodiazepines with sedative, hypnotic, anxiolytic, anti-convulsant, and anterograde amnesia properties [29] have been proven to be helpful during bronchoscopy [30]. Midazolam was commonly used in bronchoscopy because of its pharmacokinetic characteristics, but can produce long recovery times. Opioids were frequently used for bronchoscopy in combination with benzodiazepines because of their analgesic and sedative properties, and 
Fig. 2 The time course of median Modified Observer Assessment of Alertness/Sedation (MOAA/S) score in the two groups

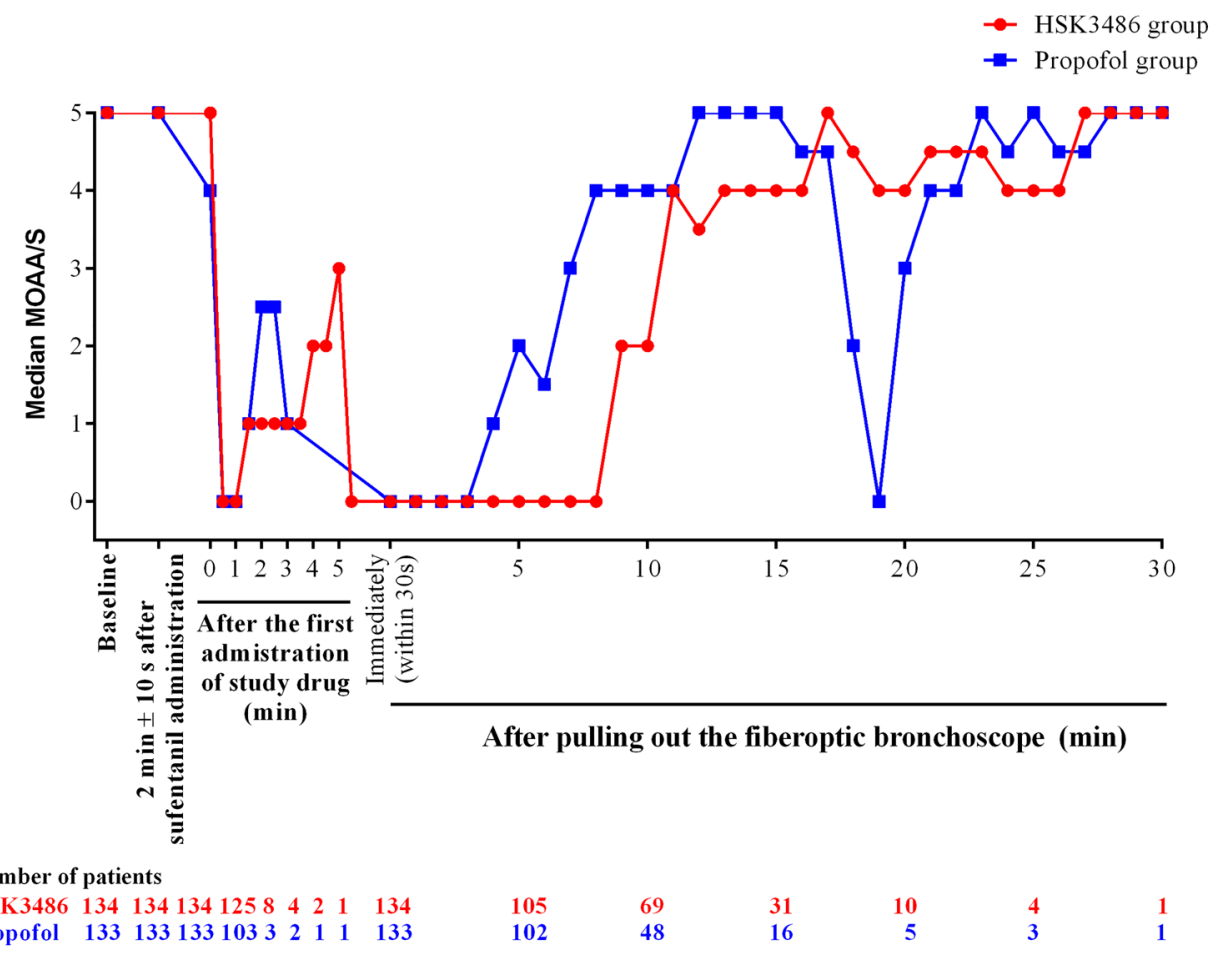

the reduction in the amount of other sedatives required [3]. However, this approach reduced the respiratory rate, resulting in ventilatory depression. Fospropofol is a water-soluble prodrug of propofol, which has been demonstrated to be safe and efficacious in patients undergoing bronchoscopy in a phase 3 randomized controlled trial [28], but fospropofol has risks similar to propofol [2]. Dexmedetomidine is a selective $\alpha_{2}$-agonist with sedative and analgesic properties. It has been shown to be safe and effective to use with flexible bronchoscopy [31] without eliciting sympathomimetic vagolytic actions, which may cause bradycardia and hypotension [32].

In the present study, HSK3486 induced, similar to propofol, a sedative/anesthetic effect with a success rate of $100 \%$. Patients treated with HSK3486 completed the fiberoptic bronchoscopy without the requirement for an alternative anesthetic/sedative and the lower boundary of $95 \% \mathrm{CI}$ for the difference (HSK3486-propofol) of success rates was $-2.8 \%$, which met the predesigned criteria of $>-8 \%$. In our study, HSK3486 had a similar duration of action and a rapid onset of anesthesia/sedation compared to propofol, both groups reaching a level of deep sedation or general anesthesia (MOAA/S $\leq 1$ ), which met the ideal sedation profiles as previously reported [27, 28]. More than half of the patients completed fiberoptic bronchoscopy without needing top-up doses in both groups. The above results indicated that the efficacy of HSK3486 $0.4 \mathrm{mg} / \mathrm{kg}$ was similar to that of propofol $2.0 \mathrm{mg} / \mathrm{kg}$.
HSK3486 had similar terminal elimination half-life characteristics to propofol, but with a potency about five times greater, and HSK3486 patients exhibited slightly longer recovery durations. The reason for this is probably the very short initial distribution half-life and high clearance rate of propofol. However, though the fully alert and recovery times were significantly longer in the HSK3486 group, it was still within 10-15 min, and the clinical implications of this delay are likely to be irrelevant. Nevertheless, a comparison of the pharmacological properties of HSK3486 and propofol will require further studies. More than half of the patients completed the fiberoptic bronchoscopy without top-up dosing, which indicated that overall anesthesia/sedation and recovery potency of HSK3486 were comparable to propofol.

A total of $172(64.4 \%)$ patients experienced 286 AEs, with $71(52.6 \%)$ patients in the HSK3486 group and 101 (76.5\%) in the propofol group. There was no grade 3 or above AEs in the HSK3486 group, and most were selfcuring or self-limited, findings similar to other anesthetics/ sedatives [27, 28, 33]. The types of AEs and drug-related AEs were similar in both the HSK3486 and propofol groups. The most remarkable difference in drug-related AEs between the two groups was pain on injection, where the incidence in patients in the HSK3486 group was significantly lower than in the propofol group. Injection pain is a common adverse reaction to propofol, which produces an uncomfortable experience for patients and increases their nervousness and anxiety. It can even elicit body movements that are not conducive to diagnosis and therapy using 
Table 3 Summary of secondary efficacy outcomes (full analysis set)

\begin{tabular}{|c|c|c|c|}
\hline & $\operatorname{HSK} 3486(n=134)$ & Propofol $(n=133)$ & $P$-value \\
\hline \multicolumn{4}{|c|}{ Time to successful induction of anesthesia/sedation, $\min$} \\
\hline Median (range) & $1.00(0.50,3.50)$ & $1.00(0.45,8.00)$ & 0.059 \\
\hline \multicolumn{4}{|c|}{ Time to fiberoptic bronchoscope insertion, min } \\
\hline Median (range) & $3.00(1.12,12.02)$ & $2.88(0.97,11.80)$ & 0.342 \\
\hline \multicolumn{4}{|l|}{ Duration of fiberoptic bronchoscopy, min } \\
\hline Median (range) & $5.78(2.12,62.40)$ & $5.78(1.67,63.67)$ & 0.972 \\
\hline \multicolumn{4}{|l|}{ Time to being fully alert } \\
\hline \multicolumn{4}{|c|}{ From extraction of fiberoptic bronchoscope, min } \\
\hline Median (range) & $8.50(0.10,27.90)$ & $6.00(0.00,29.00)$ & 0.012 \\
\hline \multicolumn{4}{|l|}{ From the last drug administration, min } \\
\hline Median (range) & $14.33(4.82,30.27)$ & $11.93(4.53,35.82)$ & 0.001 \\
\hline \multicolumn{4}{|l|}{ Time to discharge } \\
\hline \multicolumn{4}{|c|}{ From extraction of fiberoptic bronchoscope, min } \\
\hline Median (range) & $13.00(4.00,46.00)$ & $9.87(3.98,33.00)$ & 0.002 \\
\hline \multicolumn{4}{|l|}{ From the last drug administration, min } \\
\hline Median (range) & $18.82(8.82,55.17)$ & $15.93(8.50,39.82)$ & $<0.001$ \\
\hline \multicolumn{4}{|l|}{ Experimental drugs and alternative drugs } \\
\hline \multicolumn{4}{|l|}{ Total times of top-up dosing } \\
\hline Median (range) & $0(0,7)$ & $0(0,9)$ & 0.910 \\
\hline \multicolumn{4}{|c|}{ Number of patients with total times of top-up dosing, $n(\%)$} \\
\hline 0 & $71(53.0)$ & $70(52.6)$ & \\
\hline 1 & $37(27.6)$ & $38(28.6)$ & \\
\hline 2 & $14(10.4)$ & $15(11.3)$ & \\
\hline 3 & $9(6.7)$ & $7(5.3)$ & \\
\hline 4 & $1(0.7)$ & $2(1.5)$ & \\
\hline 5 & $0(0.0)$ & $0(0.0)$ & \\
\hline \multicolumn{4}{|c|}{ Times of top-up dosing during the induction phase } \\
\hline Median (range) & $0(0,1)$ & $0(0,2)$ & 0.652 \\
\hline \multicolumn{4}{|c|}{ Maximum times of top-up dosing in any 15 -min period } \\
\hline Median (range) & $0(0,3)$ & $0(0,3)$ & 0.665 \\
\hline \multicolumn{4}{|l|}{ Total dosage of top-up dosing, mg } \\
\hline Median (range) & $0.00(0.00,57.75)$ & $0.00(0.00,423.00)$ & $<0.001$ \\
\hline \multicolumn{4}{|l|}{ Total dosage of experimental drugs, mg } \\
\hline Median (range) & $26.75(14.75,79.50)$ & $142.00(26.75,549.00)$ & $<0.001$ \\
\hline $\begin{array}{l}\text { No requirement for alternative drugs, } n \\
(\%)\end{array}$ & $134(100.0)$ & $133(100.0)$ & - \\
\hline \multicolumn{4}{|l|}{ Anesthesia/sedation satisfaction scores } \\
\hline \multicolumn{4}{|l|}{ Patients } \\
\hline Median (range) & $10(6,10)$ & $10(4,10)$ & 0.341 \\
\hline \multicolumn{4}{|l|}{ Anesthesiologists } \\
\hline Median (range) & $10(0,10)$ & $10(1,10)$ & 0.972 \\
\hline \multicolumn{4}{|l|}{ Sufentanil and lidocaine } \\
\hline \multicolumn{4}{|l|}{ Top-up dosing times for sufentanil } \\
\hline Median (range) & $0(0,1)$ & $0(0,1)$ & 0.993 \\
\hline \multicolumn{4}{|l|}{ Total dosage of sufentanil, mg } \\
\hline Median (range) & $11.80(7.50,19.50)$ & $11.90(6.00,20.50)$ & 0.686 \\
\hline \multicolumn{4}{|l|}{ Total dosage of lidocaine, $\mathrm{mg}$} \\
\hline Median (range) & $0.36(0.20,0.70)$ & $0.36(0.14,0.60)$ & 0.927 \\
\hline
\end{tabular}

Data are presented as the median with range (minimum, maximum) or numbers with percentages in parentheses 
Table 4 Summary of adverse events (AEs) (safety set)

\begin{tabular}{|c|c|c|c|}
\hline & HSK3486 $(n=135)$ & Propofol $(n=132)$ & $P$-value \\
\hline Any AEs & $71(52.6)$ & $101(76.5)$ & $<0.001$ \\
\hline Grade 1 (mild) & $61(45.2)$ & $86(65.2)$ & 0.001 \\
\hline Grade 2 (moderate) & $20(14.8)$ & $30(22.7)$ & 0.098 \\
\hline Grade 3 (severe) & $0(0.0)$ & $4(3.0)$ & 0.125 \\
\hline Drug-related AEs & $50(37.0)$ & $93(70.5)$ & $<0.001$ \\
\hline Grade 1 (mild) & $37(27.4)$ & $74(56.1)$ & $<0.001$ \\
\hline Grade 2 (moderate) & $19(14.1)$ & $26(19.7)$ & 0.220 \\
\hline Grade 3 (severe) & $0(0.0)$ & $4(3.0)$ & 0.125 \\
\hline Any SAEs & $0(0.0)$ & $0(0.0)$ & - \\
\hline Sedation-related AEs & $40(29.6)$ & $42(31.8)$ & 0.698 \\
\hline Hypoxia & $8(5.9)$ & $3(2.3)$ & 0.133 \\
\hline Duration (s) & & & 0.609 \\
\hline Mean \pm SD & $180.80 \pm 172.93$ & $180.70 \pm 103.35$ & \\
\hline Median (range) & $142.00(32,555)$ & $122.00(120,300)$ & \\
\hline Hypotension & $28(20.7)$ & $36(27.3)$ & 0.211 \\
\hline Duration (s) & & & 0.520 \\
\hline Mean \pm SD & $462.80 \pm 379.78$ & $646.30 \pm 830.31$ & \\
\hline Median (range) & $390.00(120,1620)$ & $360.00(120,4800)$ & \\
\hline Bradycardia & $8(5.9)$ & $9(6.8)$ & 0.765 \\
\hline Duration (s) & & & 0.656 \\
\hline Mean \pm SD & $687.00 \pm 540.84$ & $591.80 \pm 304.93$ & \\
\hline Median (range) & $566.00(143,1560)$ & $567.00(124,1080)$ & \\
\hline $\begin{array}{l}\text { AEs related to operant reactions to fiberoptic } \\
\text { bronchoscopy }\end{array}$ & $3(2.2)$ & $4(3.0)$ & 0.976 \\
\hline
\end{tabular}

Data are given as numbers with percentages in parentheses

SAEs serious AEs, $S D$ standard deviation

Table 5 Summary of drug-related adverse events (AEs) (safety set)

\begin{tabular}{lllr}
\hline & HSK3486 $(n=135)$ & Propofol $(n=132)$ & $P$-value \\
\hline Increased serum bilirubin & $2(1.5)$ & $3(2.3)$ & 0.9798 \\
Somnolence & $2(1.5)$ & $3(2.3)$ & 0.9798 \\
Dizziness & $6(4.4)$ & $8(6.1)$ & 0.5536 \\
Hypoxia & $8(5.9)$ & $3(2.3)$ & 0.1332 \\
Hiccups & $0(0.0)$ & $1(0.8)$ & 0.494 \\
Erythematous rash & $0(0.0)$ & $1(0.8)$ & 0.494 \\
Rash & $1(0.7)$ & $0(0.0)$ & 1.000 \\
Pruritus & $0(0.0)$ & $2(1.5)$ & 0.244 \\
Cry & $1(0.7)$ & $0(0.0)$ & 1.000 \\
Pain on injection & $6(4.4)$ & $52(39.4)$ & $1(0.8)$ \\
Vomiting & $0(0.0)$ & $11(8.3)$ & 0.001 \\
Bradycardia/sinus bradycardia & $10(7.4)$ & $37(28.0)$ & 0.494 \\
Hypotension & $30(22.2)$ & & 0.823 \\
\hline
\end{tabular}

Data are given as numbers with percentage in parentheses

fiberoptic bronchoscopy. The rate of occurrence of injection pain with propofol has been reported to be about 70\% [34]. Besides other discussed causes, the drug concentration has been proposed to be a major factor for this side effect, with reduced drug concentrations leading to less pain on injection $[35,36]$, which might explain the lower incidence compared 


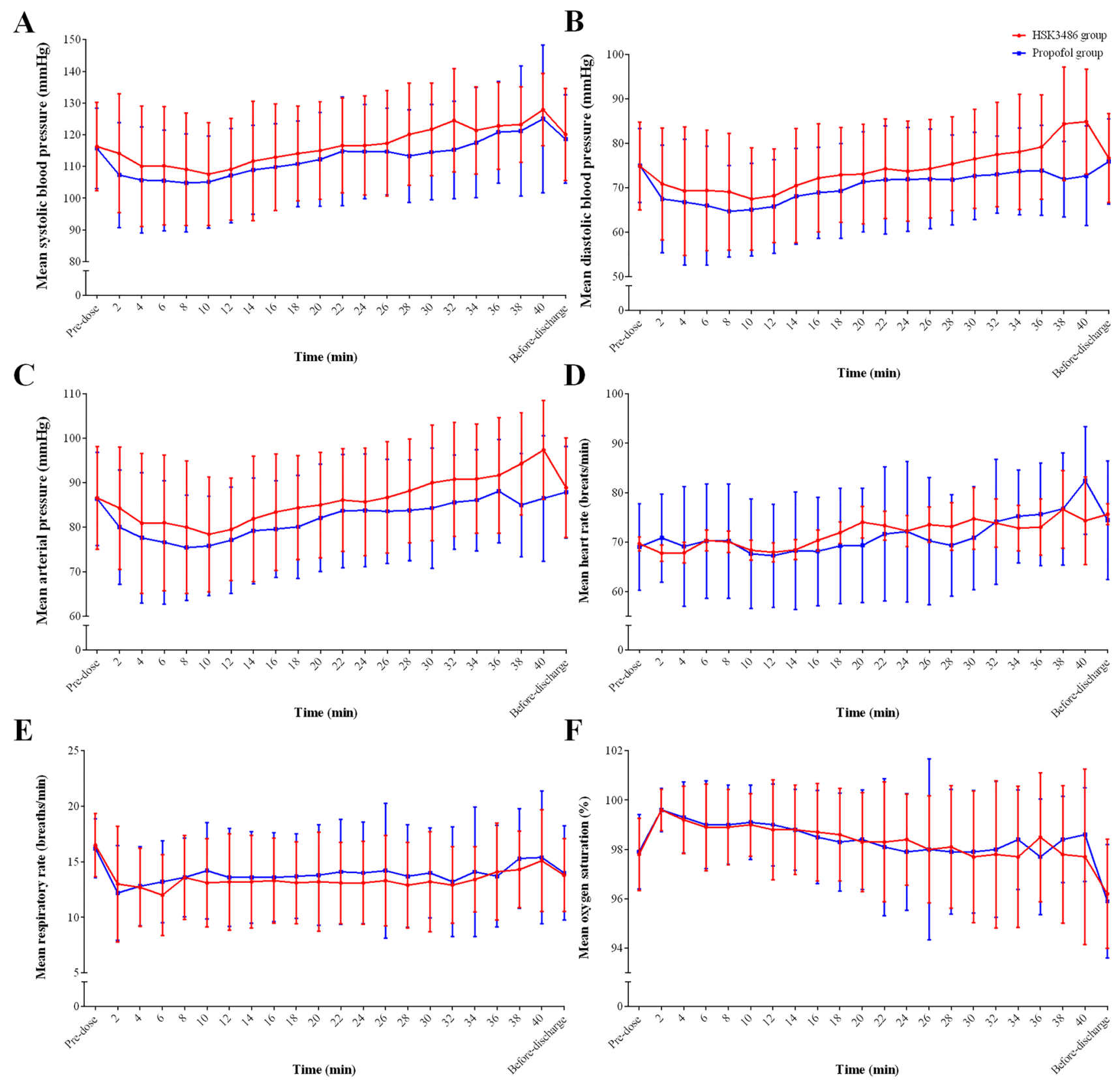

Fig. 3 Vital signs over time. A Systolic blood pressure (SBP), B diastolic blood pressure (DBP), C mean arterial pressure (MAP), D heart rate, $\mathbf{E}$ respiratory rate and $\mathbf{F}$ oxygen saturation $\left(\mathrm{SpO}_{2}\right)$

to HSK3486 administered in a fivefold lower concentration. Previous studies have noted that propofol is associated with an increased risk of hypertriglyceridemia [37], with an incidence of $27.9 \%$ in patients after propofol applications for a median time of $47 \mathrm{~h}$ [38]. However, hypertriglyceridemia after HSK3486 infusions is less likely to occur since the lipid content in essentially lower than with propofol since a $1 \%$ HSK3486 solution includes 5\% soybean oil, $2.25 \%$ glycerol, and $1.2 \%$ purified egg phosphatide versus $10 \%$ soybean oil, $2.25 \%$ glycerol and $1.2 \%$ purified egg phosphatide for a
$1 \%$ propofol solution [39], but further studies are necessary to evaluate the effects of HSK3486 on hypertriglyceridemia. Another aspect might be less environmental pollution of HSK3486 compared to propofol, since propofol has been recognized as a contributor to environmental contamination [40]. The most common sedation-related AEs, including hypoxia, hypotension, and bradycardia, occurred within 10 min after the initial administration of HSK3486 or propofol, with 40 (29.6\%) in the HSK3486 group and 42 (31.8\%) in the propofol group. HSK3486 patients exhibited a lower 
Fig. 4 Mean plasma concentration-time curve linear plot. The plasma concentration during the first $6 \mathrm{~h}$ followed first-order kinetics

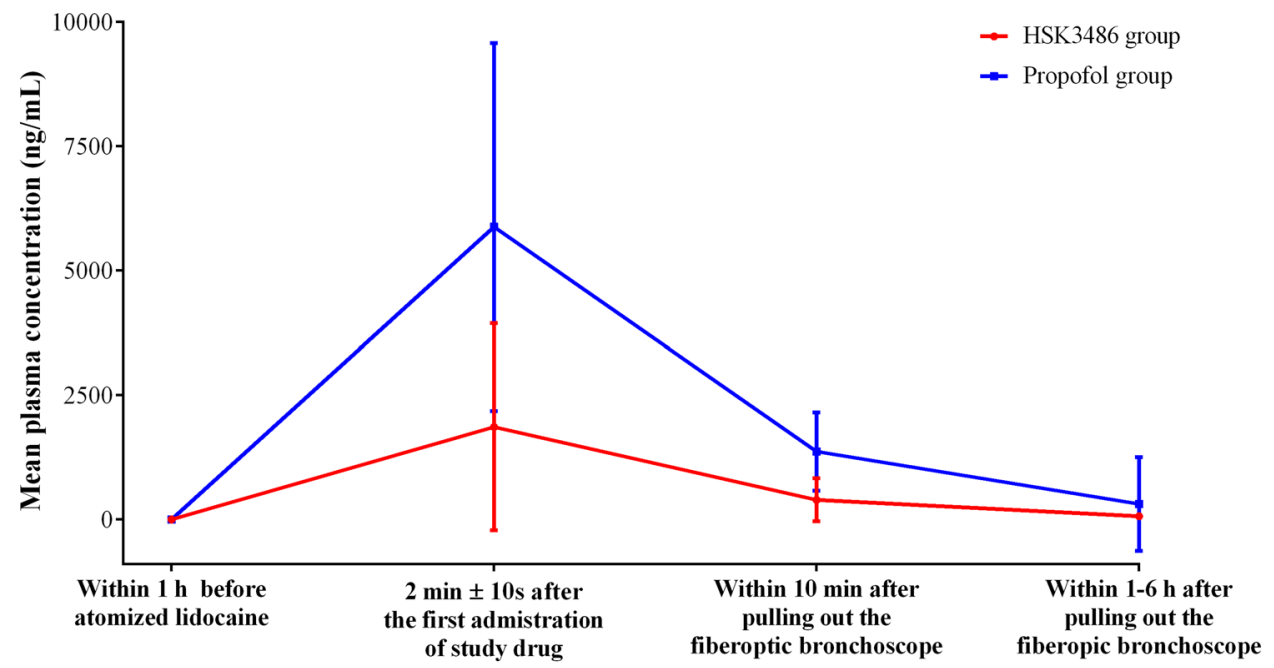

incidence of hypotension and bradycardia and an equivalent duration of hypoxia (180.80 s in the HSK3486 group vs. $180.70 \mathrm{~s}$ in the propofol group), similar to reported studies for other sedatives [41]. Hypotension is a common AE produced by anesthesia/sedation, and the underlying mechanisms still remain to be elucidated. Studies have shown that propofol inhibits myocardial contraction by reducing the concentration of free calcium ions in cardiomyocytes through a protein kinase C-dependent pathway [42]. It has also been suggested that part of the reason for hypotension caused by propofol during induction and maintenance of anesthesia is inhibition of vascular tone [43]. Vital signs and laboratory tests of patients were all stable, with no clinically significant differences between the HSK3486 and propofol groups. There was a good trend of efficacy and safety of HSK3486 in elderly patients, although the number of elderly patients in each group was small, and further studies need to be conducted to establish the routine use of HSK3486 in older patients who will undergo fiberoptic bronchoscopy.

Taken together, the present non-inferiority trial showed that HSK3486 is a useful alternative sedative drug for fiberoptic bronchoscopy with less side effects, particularly injection pain, compared to propofol. The limitations of the present study are: (1) The large proportion of patients aged $<65$ years, with 119 patients in each group, leading to a lack of guidelines for older patients, although the results are very promising. Prospective comparative studies with a larger cohort of patients aged $>65$ years will be conducted in the near future. (2) Pre-administration of sufentanil prior to fiberoptic bronchoscopy may have had an additional effect on anesthesia/sedation. To avoid this potential problem, follow-up studies will be conducted in the absence of sufentanil administration.

\section{Conclusions}

HSK3486 induced comparable anesthesia/sedation in patients undergoing fiberoptic bronchoscopy and exhibited non-inferiority to propofol, with a success rate of $100 \%$. HSK3486 had a good safety profile and a lower incidence of AEs were reported, all of severity grade 1 or 2 .

Supplementary Information The online version contains supplementary material available at https://doi.org/10.1007/s40263-021-00890-1.

Acknowledgements The authors acknowledge all investigators from the following units for their great efforts in helping us to complete the study: West China Hospital, Sichuan University; The Third Xiangya Hospital of Central South University; Sichuan Provincial People's Hospital; The Affiliated Hospital of Guizhou Medical University; The Affiliated Hospital of Zunyi Medical University; The First Affiliated Hospital of Jinan University; The Second Affiliated Hospital of Wenzhou Medical University; The First Affiliated Hospital of Wenzhou Medical University; The Second Xiangya Hospital of Central South University and Xiangya Hospital Central South University. We are also grateful to Xiaoqing Li and Xin Liu (Medkey Pharmaceutical Co., Ltd. Sichuan, China) for their excellent work in data recording and collation.

\section{Declarations}

Funding The study received funding from Haisco Pharmaceutical Group Co., Ltd. The open access fee was also paid by Haisco Pharmaceutical Group Co., Ltd. The sponsor was not involved in any of the study design; data collection, analysis and interpretation; manuscript writing and submission.

Conflict of interest ZL, HT, XZ, XW, WOY, XCW, XHZ, ZQZ, YLL, WNSG, HW, YPW and QLG declare that they have no conflict of interest.

Availability of data and material The datasets generated during and/or analyzed during the current study are available from the corresponding author on reasonable request.

Code availability Not applicable. 
Ethics approval The study was approved by the ethics committee of each participating hospital.

Consent to participate Informed consent was obtained from all individual participants included in the study.

Consent for publication Not applicable.

Authors' Contributions Conceptualization: ZL and XW; Validation: ZL, HT, XZ, XW, WOY, XCW, XHZ, ZQZ, YLL, WNSG, HW, YPW and QLG; Formal analysis: ZL, HT and XZ; Investigation: ZL, HT, XZ, XW, WOY, XCW, XHZ, ZQZ, YLL, WNSG, HW, YPW and QLG; Data curation: ZL, HT, XZ, XW, WOY, XCW, XHZ, ZQZ, YLL, WNSG, HW, YPW and QLG; Writing-original draft: ZL and HT; Writing-review and editing: XZ, XW, WOY, XCW, XHZ, ZQZ, YLL, WNSG, HW, YPW and QLG; Supervision: XW.

Open Access This article is licensed under a Creative Commons Attribution-NonCommercial 4.0 International License, which permits any non-commercial use, sharing, adaptation, distribution and reproduction in any medium or format, as long as you give appropriate credit to the original author(s) and the source, provide a link to the Creative Commons licence, and indicate if changes were made. The images or other third party material in this article are included in the article's Creative Commons licence, unless indicated otherwise in a credit line to the material. If material is not included in the article's Creative Commons licence and your intended use is not permitted by statutory regulation or exceeds the permitted use, you will need to obtain permission directly from the copyright holder. To view a copy of this licence, visit http://creativecommons.org/licenses/by-nc/4.0/.

\section{References}

1. Alon D, Pertzov B, Gershman E, Frishman M, Rahman NA, Rosengarten D, et al. The safety of laryngeal mask airwayassisted bronchoscopy versus standard nasal bronchoscopy. Respiration. 2017;93(4):279-84.

2. Wahidi MM, Jain P, Jantz M, Lee P, Mackensen GB, Barbour SY, et al. American College of Chest Physicians consensus statement on the use of topical anesthesia, analgesia, and sedation during flexible bronchoscopy in adult patients. Chest. 2011;140(5):1342-50.

3. José RJ, Shaefi S, Navani N. Sedation for flexible bronchoscopy: current and emerging evidence. Eur Respir Rev. 2013;22(128): 106 .

4. Becker HD. Bronchoscopy: the past, the present, and the future. Clin Chest Med. 2010;31(1):1-18.

5. Du Rand IA, Blaikley J, Booton R, Chaudhuri N, Gupta V, Khalid S, et al. British Thoracic Society guideline for diagnostic flexible bronchoscopy in adults: accredited by NICE. Thorax. 2013;68(Suppl 1):i1-44.

6. Gonzalez R, De-La-Rosa-Ramirez I, Maldonado-Hernandez A, Dominguez-Cherit G. Should patients undergoing a bronchoscopy be sedated? Acta Anaesthesiol Scand. 2003;47(4):411-5.

7. Hatton MQ, Allen MB, Vathenen AS, Mellor E, Cooke NJ. Does sedation help in fibreoptic bronchoscopy? BMJ (Clin Res Ed). 1994;309(6963):1206-7.

8. Goudra BG, Singh PM, Borle A, Farid N, Harris K. Anesthesia for advanced bronchoscopic procedures: state-of-the-art review. Lung. 2015;193(4):453-65.
9. British Thoracic Society Bronchoscopy Guidelines Committee aSoSoCCoBTS. British Thoracic Society guidelines on diagnostic flexible bronchoscopy. Thorax. 2001;56(Suppl 1):i1-21.

10. Putinati S, Ballerin L, Corbetta L, Trevisani L, Potena A. Patient satisfaction with conscious sedation for bronchoscopy. Chest. 1999;115(5):1437-40.

11. Matot I, Kramer MR. Sedation in outpatient bronchoscopy. Respir Med. 2000;94(12):1145-53.

12. Clark G, Licker M, Younossian AB, Soccal PM, Frey JG, Rochat $\mathrm{T}$, et al. Titrated sedation with propofol or midazolam for flexible bronchoscopy: a randomised trial. Eur Respir J. 2009;34(6):1277.

13. Lo Y-L, Lin T-Y, Fang Y-F, Wang T-Y, Chen H-C, Chou C-L, et al. Feasibility of bispectral index-guided propofol infusion for flexible bronchoscopy sedation: a randomized controlled trial. PLoS One. 2011;6(11): e27769-e.

14. Schlatter L, Pflimlin E, Fehrke B, Meyer A, Tamm M, Stolz D. Propofol versus propofol plus hydrocodone for flexible bronchoscopy: a randomised study. Eur Respir J. 2011;38(3):529-37.

15. Tan LH, Hwang NC. The effect of mixing lidocaine with propofol on the dose of propofol required for induction of anesthesia. Anesth Analg. 2003;97(2):461-4.

16. Desousa KA. Pain on propofol injection: causes and remedies. Indian J Pharmacol. 2016;48(6):617-23.

17. Marik PE. Propofol: therapeutic indications and side-effects. Curr Pharm Des. 2004;10(29):3639-49.

18. Qin L, Ren L, Wan S, Liu G, Luo X, Liu Z, et al. Design, synthesis, and evaluation of novel 2,6-disubstituted phenol derivatives as general anesthetics. J Med Chem. 2017;60(9):3606-17.

19. Bian Y, Zhang H, Ma S, Jiao Y, Yan P, Liu X, et al. Mass balance, pharmacokinetics and pharmacodynamics of intravenous HSK3486, a novel anaesthetic, administered to healthy subjects. Br J Clin Pharmacol. 2020;87:93-105.

20. Hu C, Ou X, Teng Y, Shu S, Wang Y, Zhu X, et al. Sedation effects produced by a ciprofol initial infusion or bolus dose followed by continuous maintenance infusion in healthy subjects: a phase 1 trial. Adv Ther. 2021;38(11):5484-500.

21. Li X, Yang D, Li Q, Wang H, Wang M, Yan P, et al. Safety, pharmacokinetics, and pharmacodynamics of a single bolus of the $\gamma$-aminobutyric acid (GABA) receptor potentiator HSK3486 in healthy Chinese elderly and non-elderly. Front Pharmacol. 2021:12:2232.

22. Teng Y, Ou M, Wang X, Zhang W, Liu X, Liang Y, et al. Efficacy and safety of ciprofol for the sedation/anesthesia in patients undergoing colonoscopy: phase IIa and IIb multi-center clinical trials. Eur J Pharm Sci. 2021;164:105904.

23. Common Terminology Criteria for Adverse Events (CTCAE v5.0). 2017. https://ctep.cancer.gov/protocolDevelopment/elect ronic_applications/docs/CTCAE_v5_Quick_Reference_5x7.pdf. Accessed 27 Nov 2017

24. Teng Y, Ou M, Wang X, Zhang W, Liu X, Liang Y, et al. Pharmacokinetic and pharmacodynamic properties of ciprofol emulsion in Chinese subjects: a single center, open-label, single-arm, doseescalation phase 1 study. Am J Transl Res. 2021 (in press).

25. Food and Drug Administration. Non-inferiority clinical trials to establish effectiveness. In: Guidance for industry. 2016.

26. Committee for Medicinal Products for Human U, Efficacy Working $\mathrm{P}$, Committee for Release for C. Committee for Medicinal Products for Human Use (CHMP) guideline on the choice of the non-inferiority margin. Stat Med. 2006;25(10):1628-38.

27. Pastis NJ, Yarmus LB, Schippers F, Ostroff R, Chen A, Akulian $\mathrm{J}$, et al. Safety and efficacy of remimazolam compared with placebo and midazolam for moderate sedation during bronchoscopy. Chest. 2019;155(1):137-46.

28. Silvestri GA, Vincent BD, Wahidi MM, Robinette E, Hansbrough JR, Downie GH. A phase 3, randomized, double-blind study to assess the efficacy and safety of fospropofol disodium injection for 
moderate sedation in patients undergoing flexible bronchoscopy. Chest. 2009;135(1):41-7.

29. Morgan GE, Mikhail MS, Murray MJ. Clinical anesthesiology. 3rd ed. The McGraw-Hill Companies, Inc; 2001.

30. Olkkola KT, Ahonen J. Midazolam and other benzodiazepines. Handb Exp Pharmacol. 2008;182:335-60.

31. Bergese SD, Candiotti KA, Bokesch PM, Zura A, Wisemandle W, Bekker AY. A Phase IIIb, randomized, double-blind, placebocontrolled, multicenter study evaluating the safety and efficacy of dexmedetomidine for sedation during awake fiberoptic intubation. Am J Ther. 2010;17(6):586-95.

32. Kamibayashi T, Maze M. Clinical uses of alpha2-adrenergic agonists. Anesthesiology. 2000;93(5):1345-9.

33. Oztürk T, Cakan A, Gülerçe G, Olgaç G, Deren S, Ozsöz A. Sedation for fiberoptic bronchoscopy: fewer adverse cardiovascular effects with propofol than with midazolam. Anasthesiologie, Intensivmedizin, Notfallmedizin, Schmerztherapie : AINS. 2004;39(10):597-602.

34. Picard $\mathrm{P}$, Tramèr MR. Prevention of pain on injection with propofol: a quantitative systematic review. Anesth Analg. 2000;90(4):963-9.

35. Doenicke AW, Roizen MF, Rau J, Kellermann W, Babl J. Reducing pain during propofol injection: the role of the solvent. Anesth Analg. 1996;82(3):472-4.

36. Klement W, Arndt JO. Pain on injection of propofol: effects of concentration and diluent. Br J Anaesth. 1991;67(3):281-4.
37. Devaud JC, Berger MM, Pannatier A, Marques-Vidal P, Tappy L, Rodondi N, et al. Hypertriglyceridemia: a potential side effect of propofol sedation in critical illness. Intensive Care Med. 2012;38(12):1990-8.

38. Corrado MJ, Kovacevic MP, Dube KM, Lupi KE, Szumita PM, DeGrado JR. The incidence of propofol-induced hypertriglyceridemia and identification of associated risk factors. Crit Care Explor. 2020;2(12): e0282.

39. Sebel PS, Lowdon JD. Propofol: a new intravenous anesthetic. Anesthesiology. 1989;71(2):260-77.

40. Mankes RF. Propofol wastage in anesthesia. Anesth Analg. 2012;114(5):1091-2.

41. Field-Ridley A, Sethi V, Murthi S, Nandalike K, Li S-TT. Utility of flexible fiberoptic bronchoscopy for critically ill pediatric patients: a systematic review. World J Crit Care Med. 2015;4(1):77-88.

42. Kanaya N, Gable B, Wickley Peter J, Murray Paul A, Damron DS. Experimental conditions are important determinants of cardiac inotropic effects of propofol. Anesthesiology. 2005;103(5):1026-34.

43. Nagakawa T, Yamazaki M, Hatakeyama N, Stekiel TA. The mechanisms of propofol-mediated hyperpolarization of in situ rat mesenteric vascular smooth muscle. Anesth Analg. 2003;97(6):1639-45.

\section{Authors and Affiliations}

\section{Zhen Luo ${ }^{1} \cdot$ Hong Tu ${ }^{1} \cdot$ Xiang Zhang $^{1} \cdot$ Xiao Wang $^{1} \cdot$ Wen Ouyang $^{2} \cdot$ Xinchuan Wei $^{3} \cdot$ Xiaohua Zou $^{4} \cdot$ Zhaoqiong Zhu $^{5}$. Yalan $\mathrm{Li}^{6} \cdot$ Wangning Shangguan ${ }^{7} \cdot \mathrm{Hui} \mathrm{Wu}^{8} \cdot$ Yaping Wang ${ }^{9} \cdot$ Qulian Guo $^{10}$}

1 Department of Anesthesiology, West China Hospital, Sichuan University, No. 37 Guoxue Lane, Chengdu 610041, Sichuan, China

2 Department of Anesthesiology, The Third Xiangya Hospital of Central South University, Changsha, China

3 Department of Anesthesiology, Sichuan Provincial People's Hospital, Chengdu, China

4 Department of Anesthesiology, The Affiliated Hospital of Guizhou Medical University, Guiyang, China

5 Department of Anesthesiology, Affiliated Hospital of Zunyi Medical University, Zunyi, China

6 Department of Anesthesiology, The First Affiliated Hospital of Jinan University, Guangzhou, China
7 Department of Anesthesiology, The 2nd Affiliated Hospital and Yuying Children's Hospital of Wenzhou Medical University, Wenzhou, China

8 Department of Anesthesiology, The First Affiliated Hospital of Wenzhou Medical University, Wenzhou, China

9 Department of Anesthesiology, The Second Xiangya Hospital of Central South University, Changsha, China

10 Department of Anesthesiology, Xiangya Hospital Central South University, Changsha, China 